\section{Schmerztherapeuten-Verzeichnis 1989}

Herausgegeben von J. Keller und M. Zimmermann im Auftrag der Gesellschaft zum Studium des Schmerzes für Deutschland, Österreich und die Schweiz

Das Schmerztherapeuten-Verzeichnis ist eine Zusammenstellung von therapeutischen Einrichtungen in der Bundesrepublik Deutschland. Es enthält Angaben über 140 ärztliche und 25 psychologische Einrichtungen, deren Mitarbeiter sich auf dem Gebiet der Schmerztherapie besonders weitergebildet haben. Die Angaben über Organisation und zum schmerztherapeutischen Angebot sind Selbstdarstellungen der erfaßten Einrichtungen; die Erhebungsbögen der ärztlichen und psychologischen Einrichtungen sind im Original abgedruckt und nach Postleitzahlen geordnet.

Das Schmerztherapeuten-Verzeichnis liegt jetzt in der dritten Auflage vor. Die erste Auflage erschien 1985, die zweite 1987. Die starke Nachfrage durch Ärzte, Psychologen und Einrichtungen des Gesundheitswesens zeigt, daß für das Schmerztherapeuten-Verzeichnis ein großer Bedarf besteht.

Bei der jetzt erschienenen Neuauflage sind zum ersten Mal Leistungskriterien angewendet worden, die sich an die Empfehlungen der Gesellschaft zum Studium des Schmerzes für Deutschland, Österreich und die Schweiz über die $\mathrm{Zu}$ satzausbildung zum ärztlichen bzw. psychologischen Schmerztherapeuten (siehe „Der Schmerz", Band 3, Heft 1, 1989) anlehnen. Eine Kommission der Gesellschaft hat die nachfolgenden Bedingungen für die Aufnahme in das Verzeichnis ausgearbeitet:

- Teilnahme an einer interdisziplinären Schmerzkonferenz, die mindestens einmal monatlich zusammenkommt. Zeit und Ort dieser Konferenz sollen im Schmerztherapeuten-Verzeichnis angegeben werden.

- Mindestens zwei Halbtage pro Woche Öffnungszeit für ambulante Schmerzpatienten.

- Bereitschaft, Hospitanten aufzunehmen oder/und aktiv an der schmerzbezogenen Fortbildung mitzuwirken.

- Nachweis der Teilnahme an Fortbildungsveranstaltungen $\mathrm{zu}$ schmerzbezogenen Themen mit einem Gesamtumfang von 240 Stunden während einer mindestens vier- jährigen praktisch-ärztlichen Tätigkeit.

- Nachweis der ständigen Teilnahme an der Fortbildung im Umfang von mindestens 30 Stunden pro Jahr, und der regelmäßigen Teilnahme an einer interdisziplinären Schmerzkonferenz in Höhe von mindestens 20 Stunden pro Jahr.

- Verwendung einer standardisierten Anamnese und Dokumentation von Schmerzzuständen und Bereitschaft, an einer Vereinheitlichung der Dokumentation mitzuwirken.

- Angaben darüber, welche Therapieverfahren in der schmerztherapeutischen Einrichtung praktiziert werden.

Das Schmerztherapeuten-Verzeichnis wird Ärzten, ärztlichen Organisationen, Psychologen und Behörden des Gesundheitswesens auf Anfrage zugesandt. Es ist beabsichtigt, das Verzeichnis laufend fortzuschreiben und erneut zu veröffentlichen.

Falls Sie das SchmerztherapeutenVerzeichnis bestellen möchten oder Interesse an der Aufnahme in dieses Verzeichnis haben, wenden Sie sich bitte an:

Prof. Dr. M. Zimmermann II. Physiologisches Institut der Universität Im Neuenheimer Feld 326

D-6900 Heidelberg 